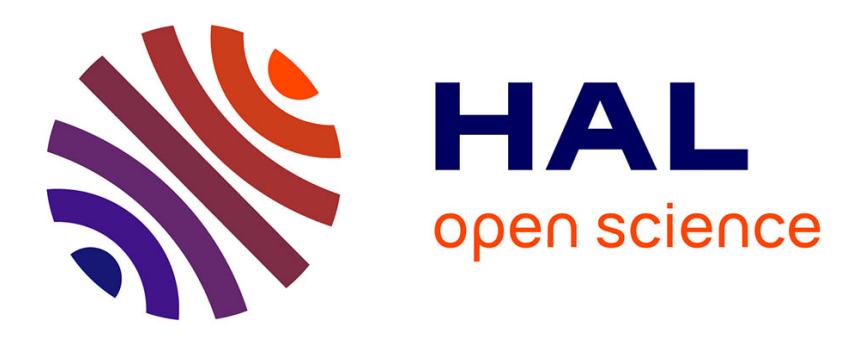

\title{
Egypte, le développement au défi du néo-libéralisme économique
}

Marc Lavergne

\section{To cite this version:}

Marc Lavergne. Egypte, le développement au défi du néo-libéralisme économique. Confluences Méditerranée, 2011, 75, pp.49-64. halshs-00558482

\section{HAL Id: halshs-00558482 \\ https://shs.hal.science/halshs-00558482}

Submitted on 3 Feb 2011

HAL is a multi-disciplinary open access archive for the deposit and dissemination of scientific research documents, whether they are published or not. The documents may come from teaching and research institutions in France or abroad, or from public or private research centers.
L'archive ouverte pluridisciplinaire HAL, est destinée au dépôt et à la diffusion de documents scientifiques de niveau recherche, publiés ou non, émanant des établissements d'enseignement et de recherche français ou étrangers, des laboratoires publics ou privés. 


\section{Egypte, le développement au défi du néo-libéralisme économique}

Marc Lavergne

\section{Chercheur au CNRS}

Directeur du CEDEJ, USR 3123 Le Caire/Khartoum

Cet article vise à proposer une appréciation du développement économique de l'Égypte qui intègre des données extérieures à la sphère économique classique. Certes, les dimensions sociales et écologiques sont aujourd'hui en voie d'intégration, encore malaisée, dans une discipline économique qui a succombé aux facilités illusoires des analyses quantitatives. L'intention n'est donc pas de porter un jugement définitif sur la santé de l'économie égyptienne, ni a fortiori sur ses perspectives d'avenir (nous vivons dans un monde si incertain que de tels pronostics devraient paraître vains, les atouts d'aujourd'hui pouvant se transformer en handicaps pour demain, tant les technologies que les cadres stratégiques évoluent rapidement).Le tableau présenté ici est donc celui de la société autant que de l'économie, et cherche à déterminer si leur relation actuelle est favorable ou non à une insertion "gagnante", pour employer la novlangue à l'honneur, dans la compétition internationale, et cela au bénéfice des Egyptiens dans leur ensemble.

Cet essai est mené à partir d'observations empiriques, mais la réalité est diverse et mal connue. Il ne s'agit donc pas de livrer un jugement global, entre aspects qui seraient considérés comme positifs ou négatifs, mais d'attirer l'attention sur la nécessité de forger de nouveaux outils d'analyse, prenant en compte la dimension psychologique et morale des politiques de développement, pour la société considérée.

\section{Introduction : une économie prospère dans un pays en crise}

\section{Des fondamentaux hors du commun}

Le premier élément à considérer, lorsque l'on s'intéresse à l'Egypte contemporaine, est la densité de sa population rapportée à l'étendue de sa superficie habitée : 85 millions d'habitants aujourd'hui sur environ $40000 \mathrm{~km} 2$ soit un record absolu à l'échelle planétaire. En dépit de la transition démographique dans laquelle l'Egypte est engagée depuis plusieurs décennies, sa population a plus que doublé au cours des trente dernières années, et devrait atteindre, si la courbe se poursuit, 140 millions d'habitants aux alentours de 2040 avant de se stabiliser ${ }^{1}$. Après une politique nataliste à l'époque de Nasser et une volonté de développer le planning familial et le contrôle des naissances sous le président Sadate, on est aujourd'hui revenu à un laisser-faire de l'Etat. La natalité est donc encadrée par deux préoccupations : l'une, inspirée par les guides religieux, les Frères musulmans et leurs épigones consiste à encourager la natalité dans une perspective de rapport de forces avec l'Occident, et au premier chef avec Israël ; l'autre es celle des contraintes du coût de la vie pour une 
population désormais aux deux tiers urbaine, qui nourrit pour ses enfants des espoirs d'ascension sociale, qui passent par l'accès à l'éducation payante.

Le deuxième élément est la nature de son économie, de type rentier, puisqu'elle repose sur le tourisme, les revenus du canal de Suez, les envois des émigrés et les recettes des hydrocarbures ${ }^{2}$. Le coton, hier encore au premier rang des exportations, suivi par le riz et d'autres produits agricoles, est aujourd'hui largement distancé (par quoi ?).

Le troisième élément est l'évolution (la « réforme ») de sa structure économique et sociale, entamée du vivant de Gamal abd el Nasser, après la défaite de la guerre des Six Jours, poursuivie par Anouar el Sadate (politique dite d'infitah, d'ouverture économique), puis par Hosni Moubarak. Cette politique a fait passer la majorité de l'économie du secteur public mis en place durant les années cinquante et soixante au secteur privé, cette privatisation causant l'apparition d'une catégorie de bénéficiaires, adeptes d'un capitalisme rentier, étroitement lié à l'appareil d'Etat ${ }^{3}$, tout en constituant un séisme social pour la majorité des Egyptiens, en particulier les paysans, les ouvriers et les fonctionnaires qui constituaient la grande majorité de la population et le socle du corps social.

\section{Une prospérité aux fondements fragiles}

L'Egypte affiche une relative santé économique, avec un taux de croissance de l'ordre de $7 \%$ annuel jusqu'en 2008, voire plus, jusqu'à la crise financière de l'automne. En partie protégée des effets de celle-ci, grâce à la faible exposition internationale de l'économie égyptienne (absence d'un système de retraites financé par les fonds de pension...), elle s'est pourtant repliée à environ 5\% en 2009, puis 4, $5 \%$ en 2010 ; mais la situation des économies méditerranéennes au sein de l'Union Européenne pourrait produire des effets de contagion dans les années à venir. Cette croissance liée à celle des revenus de rente (augmentation régulière de la manne touristique, dont l'offre se diversifie et dont les clients se mondialisent ${ }^{4}$ ), s'accompagne $\mathrm{d}^{\prime}$ un taux d'inflation préoccupant (14\% environ en $2008,12 \%$ en $2009,10 \%$ en 2010 ) et d'un taux de chômage, en particulier chez les jeunes et les diplômés, bien supérieur aux chiffres officiels, et qui toucherait au moins la moitié de la classe d'âge des 15-29 ans ${ }^{5}$.

L'économie égyptienne bénéficie donc néanmoins de ressources abondantes et diversifiées, dont témoigne la fièvre de construction et de consommation visible à travers toute l'Égypte, des banlieues de la capitale au fond des campagnes du delta, sans même parler des littoraux transformés de façon systématique en sites balnéaires réservés à une clientèle de luxé .

Mais ces ressources demeurent précaires, puisqu'elles dépendent étroitement de la santé de l'économie mondiale ou de la situation sécuritaire régionale : l’Égypte est en effet située au cœur d'une région en crise, et son secteur touristique est extrêmement volatile, face aux menaces terroristes, sans compter qu'il fluctue au gré de la conjoncturedes économies des pays industrialisés. La fréquentation du canal de Suez (4, 7 milliards de revenus en 2009-2010, en baisse de 4, 3 \% sur l'année précédente) 
est, elle, sensible aux à coups des échanges mondiaux et sous la menace des pirates opérant à l'entrée de la mer Rouge, aux larges des côtes somaliennes. Quant aux revenus des émigrés (9, 8 milliards de dollars en 2009-20107), qui proviennent pour l'essentiel des pays du Golfe, ils dépendent de leur prospérité financière, malmenée depuis 2008, et de la politique d'immigration de ces pays qui se durcit devant l'accès au marché du travail de la jeunesse autochtone, et devant la préférence accordée aux originaires d'Asie du Sud et du Sud-est, face aux originaires des pays arabes. Quant aux ressources en hydrocarbures leur avenir paraît contrasté : la production de pétrole stagne, tandis que le gaz fait régulièrement l'objet de nouvelles découvertes off shore en Méditerranée. Mais la consommation interne, favorisée par des prix subventionnés, augmente en flèche et rogne d'autant les exportations (en recul de 6, 8 $\%$ en 2009-2010).

\section{Saisir et évaluer les disparités socio-spatiales}

L'évaluation chiffrée de la répartition des revenus en Egypte ne peut se faire que d'une manière impressionniste, étant donné l'insuffisance des statistiques disponibles, qui ne prennent pas en compte les différences de modes de vie et de consommation, non plus que les ressources annexes, souvent essentielles, dans toutes les couches de la société, ni les effets de seuil qui peuvent modifier considérablement les répartitions. Mais tous les analystes s'accordent pour reconnaître sa grande inégalité. Grosso modo, les deux tiers des Egyptiens vivent au jour le jour, sans revenu assuré, et n'ont pas accès aux services de base fournis par l'Etat : éducation et santé en particulier. Un quart appartiendrait à la classe moyenne, catégorie composite culturellement et professionnellement, mais qui a accès aux biens de consommation et aux services courants (logement, éducation dans le secteur privé, accès à des soins médicaux, à un métier nanti d'un salaire permettant l'accès aux nouveaux loisirs, et de plus en plus, à l'automobile). Enfin, moins de $10 \%$ aurait un niveau de vie correspondant à des métiers qualifiés, parfois de haute technicité, liés à l'éclosion de la nouvelle économie mondialisée, ou à la haute administration, et pour une petite minorité ( $1 \%$, soit moins d'un million de personnes) de privilégiés ayant accès à une vie de loisirs et de voyages mais également à une éducation dans les meilleures universités d'Europe ou d'Amérique du Nord, après le passage par l'université américaine du Caire ou ses épigones allemande, britannique et française.

Cette répartition correspond plus à une représentation communément admise qu'à des données objectives (mais alors quid des chiffres cités plus haut ? Ne faudrait-il pas écrire alors cette représentation doit être déclinée entre la capitale et le reste du pays). Il faudrait la décliner entre la capitale et le reste du pays, Le Caire regroupant aujourd'hui le quart de la population nationale, avec une surreprésentation de pauvres venus des campagnes, ou de leurs descendants arrivés depuis plusieurs générations, mais qui demeurent cantonnés dans l'exploitation de survie des miettes de la consommation des riches, eux-mêmes concentrés dans la capitale. D'autres déclinaisons s'imposent, entre le Saïd (la vallée) et le delta, le Saïd apparaissant traditionnellement comme un cul-de sac géographique, culturel et social, coupé d'accès au monde extérieur : un angle mort de la mondialisation. Mais peut-être cette vision demanderait-elle à être révisée, non seulement du fait de l'éclosion de poches 
de prospérité dans les sites touristiques comme Louqsor, mais peut-être sous l'effet de dynamiques internes négligées, de l'impact des envois d'émigrés ou d'une volonté gouvernementale d'éradiquer les foyers potentiels d'insurrection islamiste par des projets de développement ciblés. Le Saïd n'en reste pas moins en pleine déprise démographique et économique, sous l'effet de l'émigration interne en direction de la capitale et externe vers les pays du Golfe. Il reste par ailleurs traumatisé par l'insurrection rampante des gamaat islamiya dans les années 80 et 90 et la répression féroce qui s'ensuivit et reste tributaire d'une gestion et d'un contrôle sécuritaire impitoyables ${ }^{8}$. Tout autre est le delta, qui présente lui aussi une grande diversité régionale : la pauvreté rurale y est pondérée par la proximité de grandes villes qui offrent des possibilités de pluriactivité, et par un développement rural provoqué par les envois d'émigrés. Le différentiel villes-campagnes s'y réduit beaucoup par la diffusion de petites entreprises, par la modernisation de l'activité agricole de plus en plus tournée vers le marché. Cette réorientation économique a été facilitée par la contre-réforme agraire brutale menée par le gouvernement à partir de 1995 : avec la rétrocession à leurs propriétaires antérieurs des terres nationalisées en 1952 et 1960, des millions de familles paysannes se sont vues privées de leur bien mis en valeur et entretenu depuis des générations, et de leur gagne-pain, puisque les nouveaux propriétaires citadins descendants des anciens notables ruraux, se sont massivement orientés vers des productions de haute valeur ajoutée destinées au marché ou à l'exportation, et peu exigeantes en main d'œuvre. Mais le delta se partage également entre de grandes cités industrielles appartenant au secteur d'Etat, en pleine crise économique et sociale sous l'effet des mesures de privatisation, voire de vente à des groupes étrangers, et une polarisation vers deux aires de développement complémentaires du Caire :

- l'agglomération d'Alexandrie, entourée de ses zones franches, de ses centres industriels et de ses appendices touristiques qui drainent en été la clientèle fortunée du Caire et de la péninsule arabique ;

- la zone du canal de Suez de l'autre, en plein essor avec les zones franches commerciales et industrielles de Port-Saïd et de Suez (pétrochimie, cimenterie, etc.) auxquelles s'ajoutent les nouvelles installations de Damiette (usine de liquéfaction de gaz).

La majorité de la population rurale perçoit donc des revenus annexes, difficilement comptabilisés, provenant d'une pluriactivité exercée dans la ville voisine ou sur place, de l'envoi d'émigrés à la ville ou à l'étranger.

Le nombre d'Egyptiens émigrés à l'étranger est couramment estimé à 2 millions, ce qui semble une sous-estimation grossière: on parle d'un million d'Egyptiens en Arabie saoudite, et l'on parlait déjà de deux millions d'Egyptiens en Irak durant la première guerre du Golfe (1980-1988) alors que tous les Irakiens en âge de combattre étaient au front. Compte tenu des principales destinations des migrants (Libye d'un côté, Jordanie, Irak, péninsule arabique de l'autre) et des nouvelles destinations que sont les pays occidentaux, on peut aisément doubler ce nombre. Aujourd'hui, ce sont 
donc les villes et l'étranger qui nourrissent les campagnes égyptiennes, et non plus l'inverse.

Le gouvernement égyptien encourage vivement le départ des jeunes à l'étranger, le modèle économique en vigueur ne leur permettant pas d'entrevoir de possibilité d'emploi sur place.

La structure rentière de l'économie ne repose en effet plus sur l'exploitation d'une main-d'œuvre locale, excédentaire par rapport aux besoins du tourisme, de l'industrie de transformation des ressources locales (coton, pétrole, agro-industries) ou de produits semi-manufacturés importés tels que la construction automobile ou électromécanique...) ainsi que de services marchands. Il s'ensuit une prolifération de faux emplois de services et une saturation de l'administration, alliés avec une corruption et une mendicité déguisées, omniprésentes et qui grippent la machine économique et sociale, tout en permettant aussi la survie de millions de bouches « inutiles ».

On pourrait imaginer que ces jeunes sans emploi réel puissent être formés et fournir à l'Égypte la main-d'œuvre nécessaire pour entrer dans la compétition de la mondialisation au titre de pays émergent auquel elle prétend, ayant été récemment admise comme membre du G-20. Mais le système éducatif égyptien est sinistré, les enseignants n'étant quasiment pas rémunérés et ne trouvant d'intérêt que dans des cours particuliers obligatoires, rendus indispensables par la surcharge des classes. L'Égypte a fait le choix du développement de l'enseignement privé, réservé à une élite. Mais le mal de l'enseignement en Egypte est ancien et profond : le conformisme social et culturel enraciné dans la culture nationale empêche le développement de la pensée critique, de l'intelligence individuelle au profit de la transmission par cœur des apprentissages. Cette sclérose se paie aujourd'hui, en termes de rapport au savoir, mais aussi au travail, et d'affirmation de la personnalité individuelle. Et le caractère relativement bon marché de la main-d'œuvre égyptienne ne lui permet pas de trouver sa place face à celle issue de l'Asie du Sud et d'Extrême-Orient: non seulement celle-ci s'impose à son détriment dans la péninsule arabique, mais en Égypte même, des usines privatisées licencient les ouvriers égyptiens pour les remplacer par des Indiens ${ }^{9}$, tandis que les Chinois font irruption dans le petit commerce et que l'artisanat destiné aux touristes en particulier est submergé par la pacotille importée de Chine.

A court terme, cette situation fait le jeu des milieux d'affaires et assure la pérennité d'un système politique non représentatif face à une population en désarroi, privée d'accès au savoir et à la maîtrise de son sort. Mais elle est un facteur d'instabilité et de mécontentement : on a enregistré plus de mille mouvements de grève en 2009, avec des occupations d'usines ${ }^{10}$. Cette mobilisation sociale concerne non seulement le monde ouvrier, mais aussi les catégories moyennes (agents du fisc, médecins, avocats, magistrats, corps enseignant, etc.) demandant des augmentations de salaires et l'arrêt des privatisations, le maintien des acquis sociaux et des subventions aux produits de première nécessité ${ }^{11}$. La réponse fournie par l'Etat à ces revendications contestant son orientation néo-libérale est une répression modulée: la stabilité 
sociale repose sur le contrôle sécuritaire assuré par plusieurs appareils de répression, quadrillant l'ensemble du pays ${ }^{12}$. Mais l'Etat doit cependant faire des concessions ponctuelles, étant soumis à une critique vigilante de la presse, à laquelle est octroyée un espace de liberté exceptionnel dans la région, à celle des nouveaux médias électroniques, relayés par la diaspora, et à celle des puissances étrangères soucieuses de la stabilité régionale.

\section{Un modèle de développement dualiste}

L'Etat n'est pas passif face à cette situation de fragilité. Il est porteur d'un projet de développement qui devrait permettre dans son esprit de remédier à l'impasse sociale dans laquelle se trouve le pays, et de lui ouvrir la voie d'une insertion dans le système-monde. Depuis longtemps, la solution des maux de l'Égypte est perçue par le pouvoir comme résidant dans des projets alternatifs, prenant parfois l'ampleur de mythes mobilisateurs, qui s'inscrivent dans une perspective historique à long terme. Depuis les temps pharaoniques, et derrière l'apparente l'immuabilité de l'œcoumène de la vallée et du delta, rythmée par la montée saisonnière des eaux du Nil lors de la crue estivale, l'Égypte a été le théâtre de bouleversements successifs : mise en place d'un système de bassins, puis de barrages régulateurs et creusement de canaux à partir du XIXè siècle, introduction du coton et du buffle, ouverture du canal de Suez, etc. La révolution nassérienne reste symbolisée par la construction du Haut Barrage d'Assouan et par la réforme agraire qui ont entraîné un bouleversement des modes de production agricole, ainsi que par les tentatives de colonisation du désert, à l'Ouest du delta et dans les oasis de la Nouvelle vallée. Les années Sadate ont été marquées par la prise de conscience de la menace démographique et par la volonté de capitaliser les bénéfices de la paix avec Israël en inaugurant un transfert de la population et des activités économiques hors de la capitale: conception d'un ensemble de villes-satellites autour du Caire, reconstruction des villes du canal de Suez, développement de la production pétrolière sur les rives de la mer Rouge et du Sinaï récupéré.

Le long règne de Hosni Moubarak aura permis de donner une ampleur inégalée à ces efforts et de les instituer au cœur de la stratégie économique et sociale du système.

La bonification des terres a été poursuivie dans un cadre privatisé sur toutes les marges du delta et de la vallée, au bénéfice d'entreprises et d'entrepreneurs absentéistes, voués à des productions d'exportation et à des cultures de rente : fruits et légumes, élevage hors sol... Le seul projet pris en charge par l'Etat est celui de la mise en valeur de la dépression de Tochka, en plein désert à l'extrême Sud du pays. Il s'agit dans l'esprit de ses promoteurs d'utiliser le surplus des eaux retenues dans le lac Nasser pour irriguer avec des techniques de pointe l'un des endroits les plus chauds et les plus arides de la planète. D'entrée, ce projet lancé en 1997 est envisagé comme devant être réalisé à l'aide de capitaux internationaux, pour un coût estimé par certains de 100 milliards (!) de dollars. Mais il rencontre un certain scepticisme, l'eau stockée dans le lac Nasser devant servir à compenser les crues déficitaires, et les éventuels surplus ne pouvant assurer une irrigation permanente de nouvelles 
étendues. La région retenue ne se prête en outre pas, d'un point de vue de la qualité des sols, à un usage agricole.

Entreprises d'une tout autre ampleur, et couronnées de succès, les villes nouvelles dessinées par le président Sadate ont pris corps : aux projets initiaux destinés à désengorger Le Caire, comme la cité du 6 octobre, à $50 \mathrm{~km}$ à l'Ouest, et du 10 de Ramadan, à $60 \mathrm{~km}$ à l'Est, sur la route d'Ismaïlia, ou Sadate sur la route d'Alexandrie, s'en sont ajoutées d'autres: plus près du Caire vers l'est, un arc de lointaines banlieues s'est constitué de Chorouq au Nord à Kattameya au Sud. Les promoteurs privés égyptiens ou venus du Golfe font assaut de programmes de zones résidentielles de luxe, tandis que les entreprises y ont été attirées par toutes sortes d'avantages juridiques et fiscaux. Aux banlieues résidentielles souvent closes (gated communities) viennent s'adjoindre toutes les commodités nécessaires à une vie qui exclut l'horizon de la ville traditionnelle : les universités privées, souvent de statut étranger, les banques, les sièges sociaux d'entreprises, et bientôt les administrations, dont le fameux Mogamma' qui symbolise dans l'espace et dans les esprits le centre $\mathrm{du}$ Caire, voire du pays, se délocalisent des anciens quartiers centraux d'affaires ou résidentiels devenus exigus et difficiles d'accès. Une "Smart City », à l'entrée de l'autoroute reliant le Caire à Alexandrie s'est ouverte dans un environnement totalement artificiel: immeubles de verre et $d^{\prime}$ acier au milieu de pelouses immaculées, y regroupent les sièges sociaux ou les représentations d'entreprises étrangères de haute technologie vidant le marché égyptien ou régional.

Une place à part doit être réservée au cas des Zones Franches Qualifiées, dividendes de la paix avec Israël, qui permettent de fabriquer en Egypte des produits textiles intégrant une composante (12\%) d'intrants israéliens, et leur ouvrant ainsi le marché américain.

Le modèle ne s'est pas limité au Caire, puisqu'Alexandrie, ainsi que la plupart des villes d'importance, possèdent aussi leurs villes nouvelles et leurs zones industrielles bénéficiant d'un statut privilégié. Les anciennes ceintures ouvrières, telles que Choubra el Khayma ou Hélouân, font désormais figures de villes du passé, avec leurs usines obsolètes vouées à la substitution aux importations de l'époque nassérienne, avec des technologies dépassées et $\mathrm{u}$ taux de pollution incontrôlé.

\section{Une nouvelle géographie de l'Egypte}

Le dédoublement du Caire (qui se traduit par un quadruplement en une quinzaine d'années de la surface occupée par la métropole) ainsi que des autres cités sélectionnées procède d'un constat et d'un choix: le constat de l'inutilité du «gaspillage» de fonds publics que constituerait aux yeux des dirigeants le comblement de tous les maux de l'Égypte urbaine et rurale existante, véritable tonneau des Danaïdes. Le choix assumé est donc d'affecter tous les moyens disponibles à l'édification d'une Egypte bis, sur des territoires vierges du désert et des côtes. Cette Égypte-là, sacrifiant sans état d'âme le berceau de l'Égypte millénaire, est conçue comme la locomotive qui sera en mesure de tirer les wagons de l'ancienne, sur laquelle on se contentera en attendant de fournir le minimum 
d'efforts, conservatoires et sécuritaires, pour en éviter l'explosion sociale et la catastrophe écologique : comme sur la centrale de Tchernobyl, un manteau de béton sur le feu qui couve.

L'Égypte nouvelle se compose des villes nouvelles auxquelles s'agrègent de vastes banlieues en construction, pour la classe moyenne solvable née de l'ouverture et de la mondialisation. Elles attirent désormais les fonctions commerçantes, avec les hypermarchés de la périphérie, les établissements d'enseignement huppés, et les emplois du tertiaire supérieur. Cette ceinture urbaine, au-delà des anciennes périphéries où s'entassent les pauvres, est desservie par des voies rapides, circulaires, permettant de passer de l'une à l'autre sans traverser le «vieux » tissu urbain, et de rejoindre les deux pôles extérieurs qui complètent ce dispositif: l'aéroport international, en voie de rénovation et d'agrandissement, et les stations balnéaires de la mer Rouge et de la Méditerranée. Le Sinaï est également intégré à cet espace " mondialisé », avec la station de Charm el-Cheikh, devenue célèbre en tant que centre international de rencontres diplomatiques, et résidence principale du chef de l'Etat, à l'abri des tracas du Caire.

Les moyens dévolus à ce projet gigantesque sont sans commune mesure avec ceux qui sont concédés à la gestion et au fonctionnement de l'Égypte «traditionnelle ». Les infrastructures sont en effet financées par le budget de l'Etat, et les promoteurs immobiliers comme les investisseurs bénéficient de nombreux allègements de charges. Mais la majorité des Egyptiens n'ont qu'une faible idée de la vie dans ces enclaves de rêve auxquelles ils n'ont pas accès, si ce n'est comme employés et domestiques, ou à travers les feuilletons télévisés qui les prennent pour cadre.

\section{Dans l'Égypte « intra-muros », un sentiment répandu de désarroi et d'abandon}

Pendant ce temps, les conditions de vie de la grande majorité des Égyptiens, en particulier au Caire et à un moindre degré à Alexandrie se dégradent sans rémission : le lot commun des habitants des grandes villes est le stress urbain poussé à son paroxysme, avec les embouteillages monstres, les ruptures des canalisations, les coupures estivales d'électricité, la guerre sans merci que se livrent entre eux les automobilistes - et entre eux et les piétons. Les catastrophes se succèdent, démontrant la déréliction des pouvoirs publics : incendies dévorant des bâtiments symboliques comme le Sénat en 2009, sans que les pompiers bloqués dans la circulation ne puissent intervenir, éboulement meurtrier en 2008 d'une falaise sur le bidonville de Doweiqa, qui a provoqué des affrontements jusqu'au sein du parti au pouvoir, accidents de la route et des chemins de fer, voire de bateaux, emportant chaque année des dizaines de milliers de vies humaines sans que rien ne soit fait pour arrêter l'hécatombe... Bref, cette Egypte-là livrée à la corruption alimentaire des petits fonctionnaires et d'une police pléthorique mais dépourvue de moyens, a le sentiment d'une vacuité totale de l'Etat. Le sentiment général est que celui-ci est tenu par de grands barons qui pillent d'autant plus aisément l'argent public qu'ils sont désormais puissamment installés dans les allées du pouvoir, aux côtés des dignitaires de l'institution militaire $^{13}$. 
Certes, la criminalité est encore faible par rapport à la plupart des autres métropoles d'une taille comparable à celle du Caire, mais elle s'accroît dans les quartiers pauvres, où l'Etat est quasiment inexistant. L'individu désemparé se replie sur la cellule familiale et sur la religion, voire sur lui-même, dans cette société où l'agressivité a remplacé la convivialité de naguère. La lutte pour la survie, dans les transports en commun, pour payer les factures qui s'alourdissent avec l'inflation, pour trouver les compléments de revenu indispensables à l'entretien d'une famille, pour scolariser les enfants, voire pour se marier et fonder un foyer, abolissent toute règle du jeu et toute projection dans l'avenir, donc tout souci de l'intérêt collectif. Le regain des signes apparents de pratique religieuse ne correspond pas à l'éthique sociale qui lui est traditionnellement attachée. De même, l'éthique du travail disparaît devant le souci d'efficacité et de rentabilité à court terme. Cela rejaillit sur le traitement réservé à l'environnement : la notion de bien public ou collectif, celle d'un environnement qui serait patrimoine commun, disparaissent devant une négligence due au manque d'éducation civique ou à l'exemple donné par l'Etat lui-même.

\section{Le saccage du cadre de vie et du milieu naturel}

Entre négligence de l'Etat indifférent au sort de ses citoyens, et absence de sens civique dans toutes les couches de la population, l'environnement urbain et rural se dégrade inexorablement. Quelles que soient les proclamations officielles, les programmes mis sur pied en réponse aux préoccupations concernant les biens publics mondiaux, ne sont qu'incantations sans conséquence. L'Egypte est en proie à un ravage de son environnement qui répond à sa dégradation psychologique et morale.

L'agriculture est contaminée par les produits chimiques, utilisés à haute dose et sans aucun contrôle, et en particulier depuis que le haut barrage d'Assouan a rendu les engrais chimiques indispensables, et que les rotations intégrant du fourrage ont été réduites, avec la réduction de l'élevage lié à la mécanisation ; ces polluants sont rejetés dans les drains qui se déversent dans le Nil et finalement dans la Méditerranée à travers les deux bras du fleuve et les lagunes littorales. Celles-ci, transformées en fermes à poissons, sont en voie d'eutrophisation et servent de réceptacle aux rejets toxiques des usines pétrochimiques voisines du lac Mariout près d'Alexandrie ou du lac Menzaléh près de Port-Saïd14.

Pour la plupart des habitants des campagnes, l'eau du Nil et des canaux est souvent la seule accessible, et sa consommation provoque des pathologies endémiques. Les drains sont transformés en égouts à ciel ouvert lors de la traversée des villes et des villages, cloaques pestilentiels viviers de moustiques. Si la bilharziose a reculé grâce à la diminution relative $\mathrm{du}$ nombre d'agriculteurs et à l'élévation des conditions sanitaires au cours des trente dernières années, d'autres pathologies liées à la pollution ont pris le relais : le taux de prévalence du virus de l'hépatite $C$ est de plus de $60 \%$ dans l'ensemble de la population.

L'environnement urbain est encore plus dégradé, puisque la pollution atmosphérique des grandes villes et en particulier du Caire provoque une 
généralisation des atteintes aux voies respiratoires; celles-ci devraient remettre en question l'importation et la fabrication de véhicules individuels pour un territoire aussi exigu, et inciter au développement des transports en commun. Le retard pris au Caire en matière de métro n'est cependant pas près d'être rattrapé, avec deux lignes seulement et une troisième en construction, sursaturés pour une métropole d'environ 20 millions d'habitants. Les migrations pendulaires de travail sont un calvaire quotidien pour des millions de travailleurs, et la voiture individuelle est la seule solution pour ceux qui en ont les moyens. Les extensions récentes et les villes nouvelles ne sont ainsi desservies que par des microbus, dangereux pour leurs passagers, pour les autres véhicules et pour les piétons.

Mais d'autres sources de pollution exercent un effet foudroyant, aux alentours des usines telles que les cimenteries, les usines chimiques : la ville de Hélouan rejetterait ainsi, selon une étude récente, 1000 tonnes par jour d'oxyde de carbone ${ }^{15}$. Pour être complet, il faut mentionner la plaie saisonnière du brûlage, en automne, des pailles de riz du delta, dont la fumée recouvre chaque année Le Caire de poussières asphyxiantes.

Ces fléaux ne sont pas justiciables de solutions exclusivement techniques: la complexité des interactions entre facteurs est extrême sur un terroir aussi exigu que la vallée du Nil, et nécessite des solutions prenant en compte des paramètres variés : pour prendre le dernier exemple, que peuvent faire les paysans sinon brûler ces déchets qu'ils ne peuvent stocker? L'Etat a certes favorisé la construction de quelques usines d'agglomérés végétaux qui pourraient valoriser ces déchets, mais où sont les moyens de transport nécessaires ? Bref, ces problèmes sont d'abord des problèmes de choix politiques et sociaux, donc de gouvernance, au-delà de leurs aspects de compétence ou d'organisation.

\section{Désengagement contre développement?}

Le désengagement de l'Etat est donc modulé, d'autant que le personnel politique, parlementaires, ministres et président, sont des acteurs économiques de premier plan, tout comme l'armée qui a édifié un complexe économique couvrant l'ensemble des secteurs productifs, et qui assure des revenus à sa haute hiérarchie : ce système militaire n'est pris en compte dans aucune statistique, ni en ce qui concerne sa ponction en amont sur les ressources de l'Etat, ni en aval en ce qui concerne la distribution de ses revenus.

Retrait donc de l'Etat de ses responsabilités en matière de régulation sociale, d'éducation et de santé, d'infrastructures «classiques » et de respect de la loi, mais maintien de son rôle central dans le projet économique d'intégration au système monde, à travers des investissements lourds dans les infrastructures liées à l'attractivité internationale, et dans la dévolution des ressources disponibles entre les acteurs de la nouvelle économie. Un système où le roi du fer à béton, Ahmed Ezz, est le secrétaire général du parti au pouvoir, et a pu à ce titre faire respecter son monopole dans ce domaine, et manipuler à sa guise les cours de ce produit vital pour $l^{\prime}$ économie nationale ${ }^{16}$. 
L'Etat porte donc une responsabilité déterminante dans la dégradation des valeurs individuelles et sociales. La majorité des Égyptiens sont dans un état moral oscillant entre abattement et révolte devant l'ampleur des maux qui les accablent. Plus grave sans doute, le délitement de la société ouvre le champ à l'emprise de comportements et d'attitudes individuelles délétères : le stress urbain entraîne agressivité, dédain à l'égard des normes de fonctionnement régulé de la société, mépris pour l'environnement et le cadre de vie, et cupidité fonctionnant comme une boussole des relations sociales. Paradoxe d'une société en voie de réislamisation rapide et spontanée, au moins dans les comportements sinon dans la spiritualité, jamais le matérialisme n'a été aussi prégnant en Egypte. La quête de l'argent, à tous prix, y compris par les moyens les plus réprouvés, à tous les niveaux de l'échelle sociale, est devenue le déterminant des comportements individuels, bousculant les valeurs sociales, morales et même familiales. Elle manifeste l'émergence de l'individu, mais au détriment de toute vision collective à long terme.

Il faudrait certes nuancer ce tableau, qui s'applique principalement à la capitale, la campagne et les villes de province conservant un tissu de relations familiales et sociales qui structure encore les mentalités. Mais c'est la capitale qui donne le ton, qui concentre les pouvoirs, et qui émet ses valeurs à travers les feuilletons télévisés et les scandales qui émaillent la vie politique et économique du pays.

\section{Quel impact sur la compétitivité et l'attractivité internationales de l'Égypte?}

La question centrale que pose l'évolution de l'Egypte depuis sa réouverture économique à tout va au monde extérieur n'est pas la validité de tel modèle par rapport à tel autre : que le secteur privé soit le moteur de l'économie est admis par à peu près tout le monde aujourd'hui, et la preuve est faite que l'intérêt des entrepreneurs et des investisseurs est le plus sûr moyen de l'innovation et de la compétitivité. Mais dans un système à la fois centralisé et rentier, cet esprit d'entreprise est biaisé par la capacité différentielle d'accès à ces rentes, en fonction de la proximité du pouvoir politique.

D'autre part, il s'agit d'un système malthusien, qui se refuse à maximiser les ressources humaines pour conserver les clés du pouvoir et de la richesse, en investissant dans les secteurs sociaux comme l'éducation et la santé. Faute également d'un système politique ouvert, l'attraction des capitaux étrangers ne peut se faire qu'en mettant sur le marché, à bon compte, des ressources non-renouvelables et des biens communs. Le néo-libéralisme n'est en l'occurrence pas conciliable avec une politique de développement, lorsqu'il aboutit à un désengagement total de l'Etat et à l'abandon de tout souci de l'intérêt de la communauté, et donc de durabilité, et ne repose in fine que sur un appareil sécuritaire et sur l'adhésion de l'armée au système. Et l'on peut se demander si ce démaillage de la société, la confusion mentale qui en résulte, la multiplication des comportements déviants et délinquants, sur fond d'injustice sociale, de vénalité et de soumission des instances de gestion et de régulation, n'ont pas un impact sur les perspectives de développement. Dans un 
univers mondialisé, la comparaison entre nations ne porte pas que sur les grands agrégats économiques, ou sur la disposition de ressources tangibles. Elle repose également sur des facteurs sociaux et humains. Certains sont d'ores et déjà pris en compte par l'indice de développement humain, mais celui-ci mesure plus des résultats que des potentiels. Parmi ceux-ci, le cas égyptien montre l'importance qu'il faudrait accorder aux aspects psychologiques et moraux, signes de la santé d'une nation, et gage de sa capacité de mobilisation pour un progrès partagé. 